This item was submitted to Loughborough's Research Repository by the author.

Items in Figshare are protected by copyright, with all rights reserved, unless otherwise indicated.

\title{
A simulation module for supporting the manufacture of high value added electronics manufacturing
}

PLEASE CITE THE PUBLISHED VERSION

PUBLISHER

(c) IEEE

VERSION

VoR (Version of Record)

\section{LICENCE}

CC BY-NC-ND 4.0

\section{REPOSITORY RECORD}

West, Andrew A., Lina A.M. Huertas-Quintero, Diana M. Segura-Velandia, Paul P. Conway, David C. Whalley, Antony R. Wilson, and Radmehr P. Monfared. 2019. "A Simulation Module for Supporting the Manufacture of High Value Added Electronics Manufacturing”. figshare. https://hdl.handle.net/2134/4215. 
This item was submitted to Loughborough's Institutional Repository (https://dspace.lboro.ac.uk/) by the author and is made available under the following Creative Commons Licence conditions.

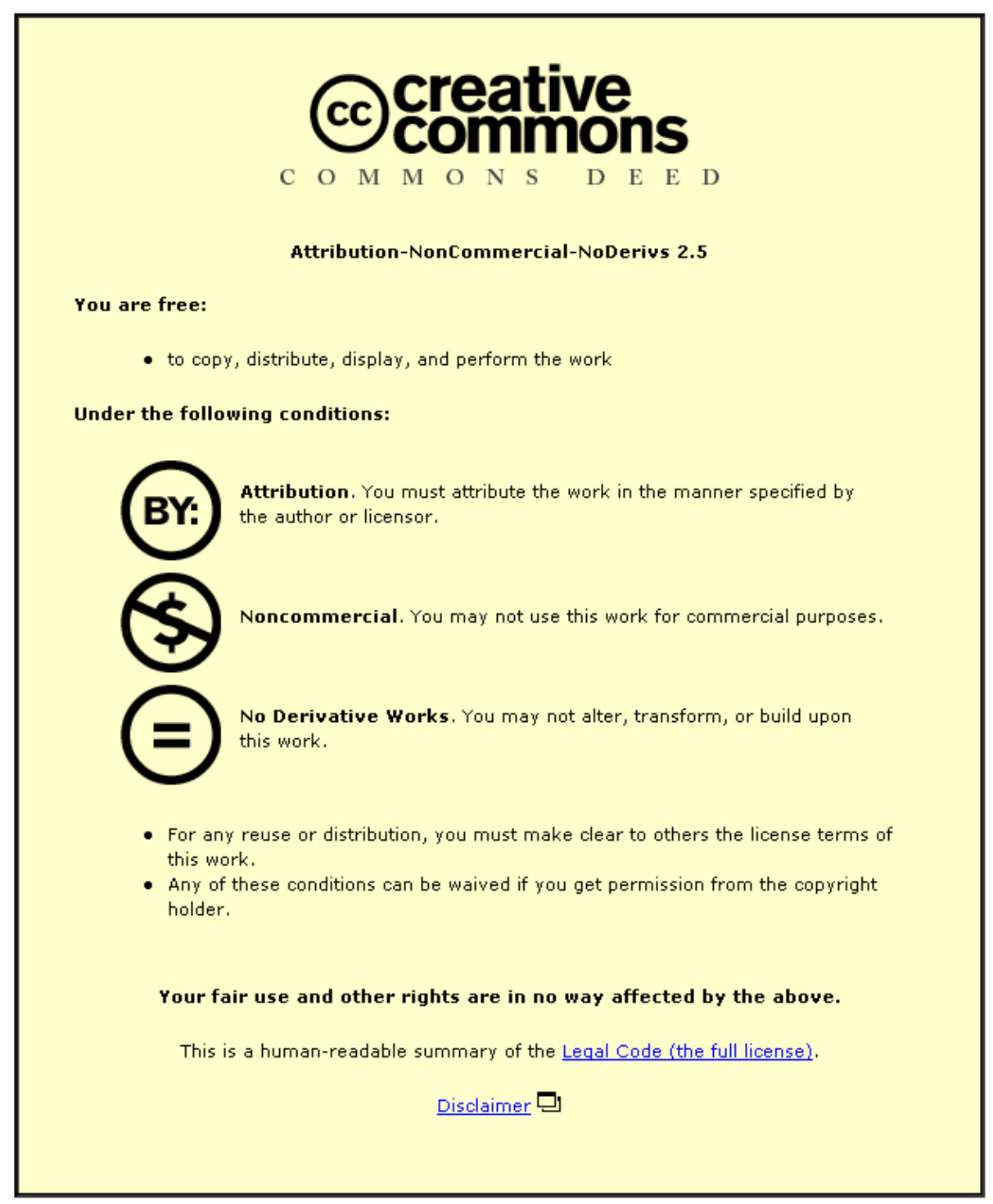

For the full text of this licence, please go to: http://creativecommons.org/licenses/by-nc-nd/2.5/ 


\title{
A simulation module for supporting the manufacture of high value added electronics manufacturing
}

\author{
L. A. M. Huertas Quintero, A. A. West, D. M. Segura Velandia, P. P. Conway, D. C. Whalley, A. Wilson, R. Monfared. \\ Wolfson School of Mechanical and Manufacturing Engineering, Loughborough University \\ Loughborough, Leicestershire, LE11 3TU, UK \\ L.A.Huertas@lboro.ac.uk
}

\begin{abstract}
Given the global pressures and demanding requirements for high value added electronics manufacturing, it is vital to make the right decisions on the shop floor. One of the main shop floor level decisions in the domain is the selection of the most appropriate scheduling strategy for the available manufacturing system. Simulation has proved to be a powerful decision support tool. However, very few studies have used this potential to support the evaluation of scheduling strategies in a manufacturing context. A component-based simulation tool to evaluate the performance of scheduling strategies on a particular system is presented in this paper. The component based structure of the simulation tool allows the main problem requirements to be addressed. An example, based on a real company, illustrates the nature of the simulation results and the kind of support that can be obtained.
\end{abstract}

\section{Introduction}

Global pressures (see Figure 1) have forced western electronics manufacturers to focus on high value added production, as well as to constantly introduce new designs and adopt new technologies. This situation generates a highly dynamic environment in which changes must be quickly undertaken, whilst maintaining or improving the main customer requirements in the domain i.e. low lead-time, low cost, high quality and high reliability. Computer support system plays an important role in allowing such companies to respond with agility.

A common issue in the high value added electronics manufacturing domain is the poor performance of planned job shop schedules [1]. Some techniques usually practiced in the industry for scheduling are oversimplified and hence, ineffective. Different methods have been explored in the dynamic job shop scheduling literature aiming to solve this problem [1-5].

Given the number of scheduling strategies that has been developed in recent decades, the evaluation of scheduling strategies has become vital for decision makers to be able to select the best strategy for a particular manufacturing system. Very few publications have focused on the solution of this issue. Some authors have proposed evaluation frameworks, but none have focused on presenting a simulation structure that is suitable to satisfy the requirements of the problem.

A component based simulation tool that aims to fill this gap is presented in this paper. The tool enables the user to evaluate job shop scheduling strategies and support decision makers in selecting the best strategy.
The paper is organised as follows. The first section gives a description of the high value added electronics manufacturing domain, its characteristics and its needs. The following section presents the background regarding job shop scheduling, scheduling strategies and the need for tools that enable the evaluation of these strategies. A brief review of previous research that has focused on this need is also given. Later on, a software tool is proposed that focus on the simulation structure perspective to facilitate the evaluation of scheduling strategies. This is followed by an example based on a UK located electronics manufacturing enterprise in order to illustrate the kind of results provided by the tool proposed. Finally, some conclusions regarding the work presented are listed, and a brief account of the future prospects is given.

\section{High value added electronics manufacturing}

Over recent decades, manufacturing companies have had to relocate and adapt to new environments to take advantage of their strengths. In the case of electronics manufacturers in Western Europe, high volume manufacturing has been migrating to low wage regions. The companies that maintained manufacture in Western Europe have typically specialised in high value added products. In this new environment, production is usually characterised by low volume orders and high product variety.

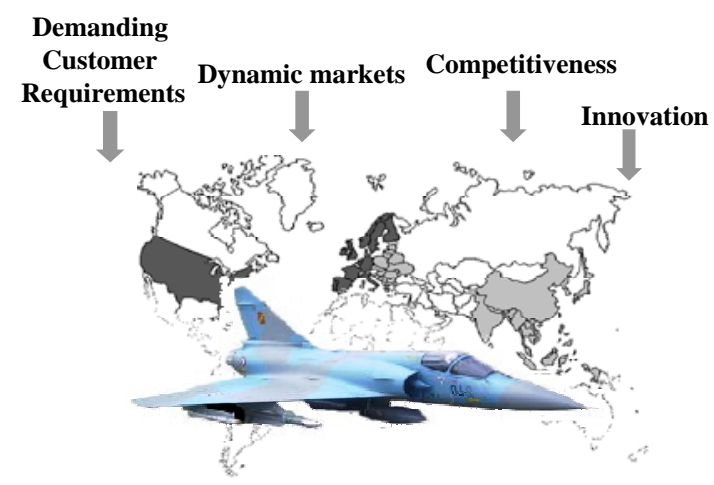

Figure 1 Global pressures on the domain

These features, as well as the global economic behaviour, generate pressures on these high value added electronics manufacturers. Some of these pressures, illustrated in Figure 1, are increased competition, dynamic markets, government legislation and the need for fast innovation. These elements make part of a challenging environment for the industry in the domain. In addition, customers particular to this industry sector 
usually impose demanding requirements that must be met (i.e. low lead times, low cost, high quality and high reliability).

To be able to endure and succeed under these conditions, companies need to make effective decisions, and adopt successful strategies. Decision support tools are vital to improve the understanding and visualisation of manufacturing systems, and to evaluate the performance of proposed schemes. Simulation has been widely recognised as a powerful decision support tool, both, in the industrial and the academic context. The initial model needed to build up the simulation usually provides the understanding and visualisation aids. The actual implementation of these models (i.e. simulation) provides the evaluation and prediction functionalities, greatly reducing the risk of implementing changes in the actual system. From this point of view, simulation tools are essential to make the right decisions in order to adopt a manufacturing system to external requirements.

Production planning and control decisions are fundamental in the high value added electronics manufacturing domain. These two activities are the ones that enable a feedback cycle in the system (i.e. control) and determine the production characteristics taking into account the external requirements and any system disturbances (i.e. planning). Hence, the requirements can be satisfied and also continuously improved. One of the most important decisions in this framework is the selection of an appropriate scheduling strategy. The importance of this decision is related to the criticality of the role of schedules in manufacturing systems in this domain. This issue is discussed in greater detail in the following section.

\section{Job shop scheduling}

Scheduling is a critical activity in high value added electronics manufacturing. The manufacturing systems in the domain usually behave as job shops i.e. utilising various machines, and where various jobs are produced by different sequences of these machines. As represented in Figure 2, job shop scheduling consists of assigning each of the jobs to the appropriate machines, such that a particular performance criterion is met, such as minimisation of job tardiness [6].
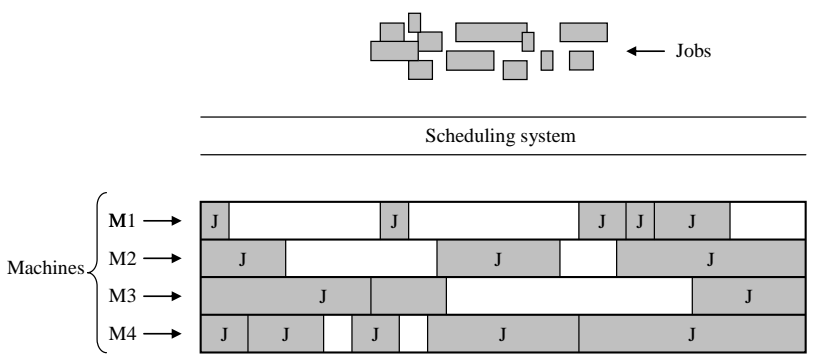

\section{Figure 2 Job shop scheduling representation}

It is common practice in the industry to generate schedules manually, or using simple tools such as Gantt charts. This kind of technique lacks predictive capability and is therefore not appropriate given the complexity and dynamicity of the manufacturing process in the domain (e.g. random arrival time and stochastic processing times) [7].Ineffective schedules generated using badly chosen methods can have harmful effects on the products and the processes (e.g. low resource utilisation, increased product cost and even poor quality in some cases).

Different methods have been explored in the dynamic job shop scheduling literature (refer to review papers [8, 9] for examples) aiming to solve this problem. The proposed techniques can be classified as dispatching rules, mathematical methods, neighbourhood search methods and artificial intelligence techniques. All of these categories are characterised by different advantages and drawbacks [8]. Some of them require little effort to implement making them practical for industry to apply, but oversimplify the problem (e.g. dispatching rules). Others take into account the detailed specifications of the manufacturing system, but its implementation demands considerable effort and computational capacity (e.g. mathematical techniques). Many studies in the literature have proved the success of one of these techniques for different types of manufacturing systems, under different performance criteria [1-5]. However, no one single technique is the best and the success of a particular strategy strongly depends on the specifications of the system in which it is to be implemented.

For the above, it is clear that companies need to evaluate the performance of scheduling strategies for their own manufacturing system, and take into account their own goals. Only a few studies have aimed to support the solution of this issue. In general, they include a simulation engine as the evaluation entity of the solution. Most of the proposed approaches focus on the schedule performance analysis, once the performance results are provided by the simulation. One of the earliest studies is presented in [6], where a design of experiments (DOE) approach is used to evaluate flexible manufacturing systems (FMS) performance with reduced computational effort. The effect of various operating parameters, including scheduling policies, is measured using a set of standard performance criteria. Later on Mignon, et al. proposed in [7] a simulation engine to evaluate the impact of uncertainty on different schedule strategies. The simulation engine consists of a Monte-Carlo simulation and the simulation software BATCHES [8]. The authors proposed two meaningful metrics for the evaluation of scheduling strategies in uncertain systems i.e. robustness and performance.

Almost a decade later, Cha and Jung [9] combined a simulation model with a fuzzy logic engine to evaluate different scheduling rules. Quantitative values of different performance criteria are combined with qualitative importance indices to provide a general performance metric. A similar methodology was presented by Chan and Chan in [10] to evaluate the quality of schedules. The importance of scheduling strategies evaluation for decision-makers, and the relationship between the evaluation and the general aims or policies in a company, 
are pointed out by Chan and Chan. One of the most recent studies that consider the problem of evaluating scheduling techniques is [1], which identifies scheduling evaluation tools as a primary need in the industry. The system presented for the evaluation of scheduling solutions consists of a user interface that enables the user to input the information regarding the particular manufacturing system to be processed, a virtual library to store past test cases, and an emulator system to test the scheduling proposals.

Although important progress has been achieved, existing studies are still weak from the simulation point of view. Most of them focus on user interface or on the posterior analysis of the performance metrics provided by the simulation. However, the interest on the development of a simulation structure that suits the industrial requirements has been poor. A more detailed study regarding the development of a simulation tool that is appropriate to support the evaluation of scheduling strategies is needed. The research presented in this paper aims to tackle this need.

\section{Simulation module for scheduling evaluation}

A simulation tool to evaluate job shop scheduling is presented in this paper. The simulation enables the user to evaluate the performance of different scheduling strategies for a specific manufacturing system. Quantitative metrics based on the domain customer requirements (i.e. time and cost) are provided to the decision maker. The information provided is a valuable aid for the selection of the most suitable scheduling strategies according to the goals of a company. The main differentiator of the simulation tool proposed is a component based structure that matches with the objects in a real manufacturing system in the domain, and that provides a hierarchical composition that enables different levels of detail. The composition of the simulation permits tackling important requirements of the domain such as low model development times and the capability of implementing complex models that accurately represent the systems proposed. The simulation structure (i) facilitates the integration of scheduling strategies to be evaluated, (ii) enables modularity and reusability, (iii) requires low levels of software expertise to implement models and (iv) permits high levels of detail and complexity in the models.

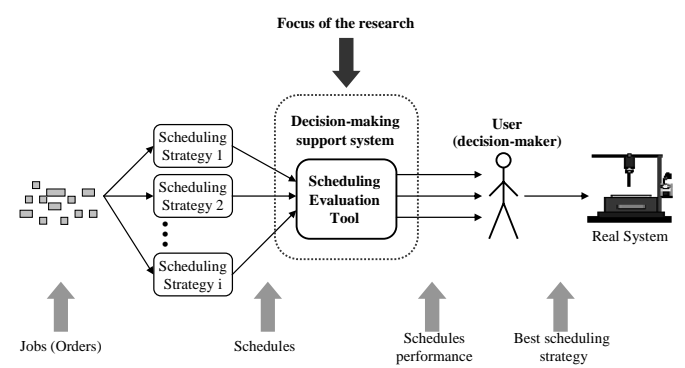

Figure 3 Role of simulation tool in the shop floor
Some companies have well established and effective strategies. More dynamic companies are usually searching for new strategies that fit new manufacturing conditions. In this case, different scheduling strategies to control the production on the shop floor are considered. This situation represents the context where the deployment of the simulation tool proposed would be most valuable.

Once the simulation model for the manufacturing system to be evaluated is developed, each scheduling strategy would generate a different schedule for a set of orders. As shown in Figure 3, these schedules are the input of the model and the simulation is executed to determine the performance of the system for each schedule. The comparison of the performance values provided determines the best scheduling strategy according to the system and its specifications. Additionally to the comparison, the performance values could be further analysed using different techniques or tools (e.g. a fuzzy methodology presented in [10], which compute quantitative performance values obtained from simulations and qualitative goals and policies from the companies to provide a more meaningful evaluation). Finally, the best scheduling strategy would be implemented into the real shop floor.

The methodology that has been followed to deploy the proposed simulation tool can be divided in three main steps: (i) simulation model development, (ii) scheduling strategy introduction and (iii) simulation execution. These steps correspond to the actual deployment of the simulation tool. In order to effectively complete the support process, three additional steps are suggested (iv) results analysis and (v) knowledge capitalisation. These steps are described as follows.

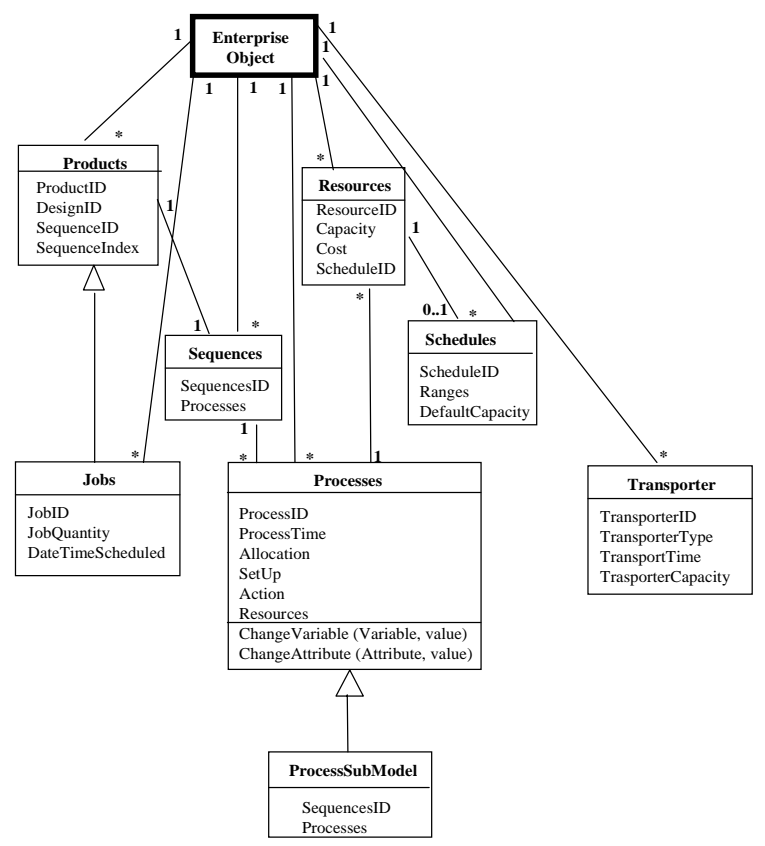

Figure 4 Component-based simulation structure 
This structure, illustrated in Figure 4, has two characteristics that are important. The first one is its component based nature. The simulation is defined by components or constructs that match with the real objects in a manufacturing system. The main components are illustrated in Figure 4. This structure enables the user to easily identify the components of the system to be modelled and represent them in terms of simulation constructs. With these components, simple models can be generated in short times and with relatively low effort. The second is the use of "sub-model" components, which enables the user to build more complex and detailed models.

The introduction of the scheduling strategy as an input can be performed in three ways. The first is when the simulation strategy is implemented as an algorithm in a programming language. In this case, the algorithm can be integrated to the simulation through an interface coded using a general programming language (e.g. Visual Studio .NET). The schedule automatically generated by a piece of code is translated into attributes of the simulation model and the simulation executed. The second option is to implement the scheduling strategy independently from the simulation tool, and then entering the schedule information manually into the model. The last alternative is to run the simulation model with the default values (i.e. all the orders are released at the beginning of the simulation and they follow a first in first out rule (FIFO) from there on).

Once the simulation model is completed and the scheduling strategy input is ready, the simulation can be executed. After the simulation has finished, the results should be analysed by the decision maker. At this point the user should be able to select the best scheduling strategy for the system under study, according to a particular criterion and the company goals. In addition, the simulation tool offers the option of saving the simulation model generated as well as the results obtained. The usage of this option enables the user to capture the knowledge generated and reuse it to support future decisions.

\section{Pilot execution}

An example based on a high value electronics manufacturing company located in the UK, is presented. The company that has been simulated is a PCB manufacturing subcontractor. They produce a high variety of products for different applications, including the aerospace and defence sectors. The model of the manufacturing system was constructed based on information gathered from the company information systems and several interviews with the shop floor personnel.

Just one scheduling strategy was evaluated, which corresponds to the current practice within the company studied. This strategy, as many others in the industrial environment, is based on Gantt charts and some rules specific to the domain. These rules are mainly related to the machines capability. In this case, the scheduling strategy was manually integrated. The scheduling strategy was implemented by the company and the resulting scheduled information was entered manually as part of the simulation model. The results produced after the simulation execution, are given in terms of job tardiness, lead time per job and resource utilisation. The most important results are illustrated in Figure 5, Figure 6 and Figure 7, where PL1, PL2, PL3 and PL4 are the designators of the four production lines in the simulated manufacturing system.

Figure 5 illustrates the lead time (y-axis) per job (xaxis), classified according to the impact on the product (i.e. value added, non value added, waiting and quality). The first important fact that can be identified from this figure is the fact that all the jobs spend most of the time in the shop floor waiting to be. Further examination of the results showed that this waiting time corresponded mainly to set up time (i.e. $88 \%$ of the waiting time per product is due to set up processes). Job tardiness values also seem to be very high (i.e. approximately one month in some cases). Moreover, if waiting times are plotted against job tardiness, as in Figure 6, a tight relation can be identified. When the results by process are analysed, one of the processes can be identified as the cause of high waiting times, and so, the cause of high job tardiness. The process is an inspection processes, and just one inspection machine is available for all the jobs coming from four production lines, hence creating a bottleneck. Figure 7 illustrate the utilisation of each resource (i.e. employees and machines) during the simulation. The values obtained verify this conclusion, showing that the inspection machine utilisation is remarkably high when compared to the utilisation of the other resources. A generally low utilisation of the resources available is also evident from Figure 7.

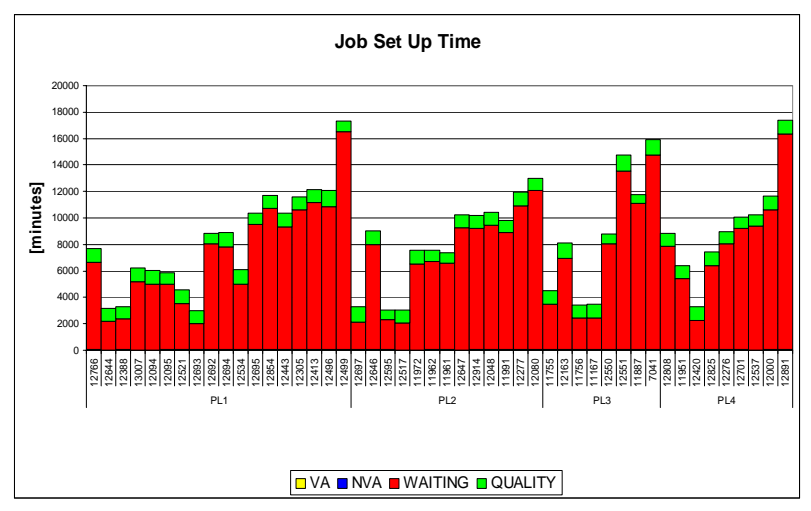

Figure 5 Setup time per job 


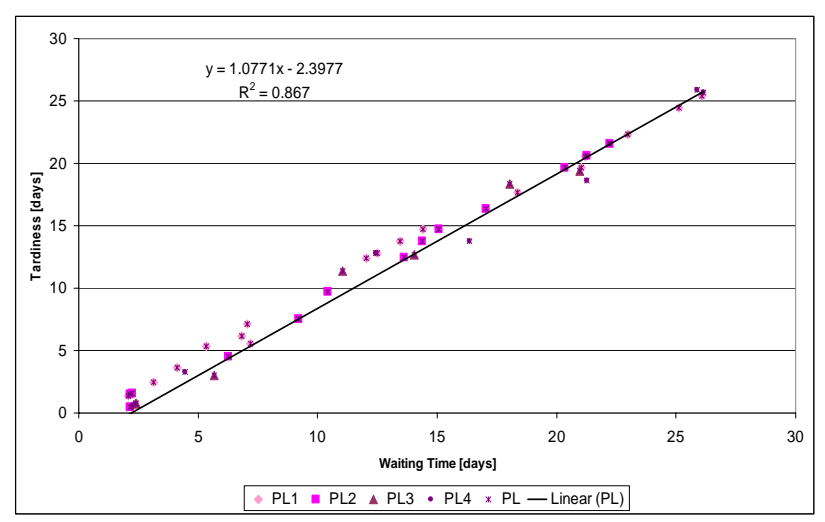

Figure 6 Waiting time vs. Tardiness

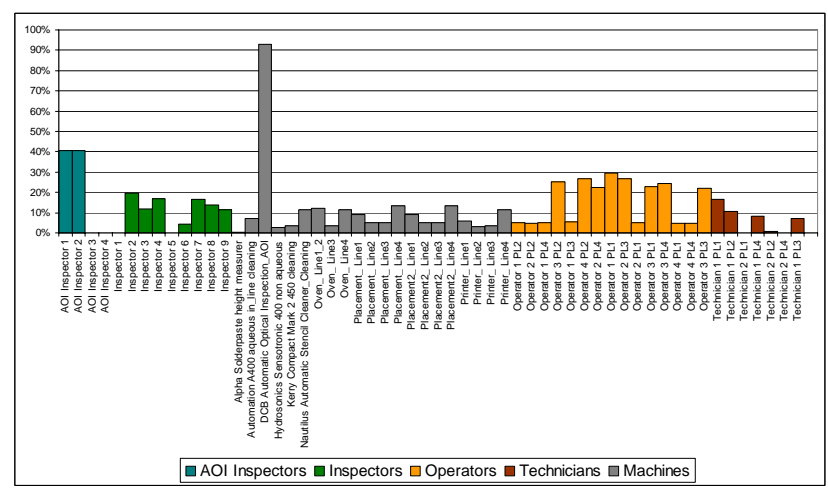

Figure 7 Resource utilisation

These results show examples of how the performance of a manufacturing system can be represented for a particular scheduling strategy, and how they might be useful. Given that these results correspond to a pilot run, the full potential of the simulation tool cannot be demonstrated in this example. However, it must be taken into account that the main advantage of the results given by the simulation tool will be in comparative terms with other scheduling strategies, and even other manufacturing system architecture scenarios.

Work of the research group is ongoing to refine of simulation structure, and to generate representative results that provide evidence of the full potential of the tool. In order to generate this evidence, different scheduling strategies will be evaluated for various scenarios based on the industrial partners of the projects involved. The simulation will provide a measure of the evaluated strategies in terms of time, cost and resource utilisation. From these results, it is expected to identify the best type of strategy for each scenario and obtain a better understanding of the relation between the performance of different scheduling strategies and the characteristics of a manufacturing system.

\section{Conclusions}

Over recent decades, global markets and economic conditions have imposed substantial pressures on high value added electronics manufacturing companies. To be able to respond to these pressures whilst satisfying time demanding customer requirements, the right decision must be implemented. Software tools and simulation in particular, are vital to support decision making processes in such a complex domain.

One of the most important decisions in the domain is the selection of the best scheduling strategy to plan the job execution order. However, very few software tools have been developed to support this decision. In this document a simulation tool is presented, that supports the evaluation of scheduling strategies. The simulation enables users to evaluate different scheduling strategies providing quantitative metrics of its performance and provides valuable information to decision makers. An example based on a high value electronics manufacturing company located in the UK, is presented. The real scheduling practice is modelled and implemented using the simulation tool. The example demonstrates how the tool can be used and the preliminary results generated prove the usefulness of the tool.

Future work will be directed towards refining the simulation structure proposed and evaluating different scheduling strategies for the domain studied. A long term aim of the research is the extension of the simulation to other applications (e.g. quality, process control) and other manufacturing domains. The refinement of the component based structure and the integration of physical models to the simulation tool are essential in achieving these objectives.

\section{Acknowledgments}

The authors wish to express their gratitude to their industrial collaborators in the CLOVES and DISCOVER projects. This work was supported financially by the Innovative Electronics Manufacturing Research Centre (IeMRC).

\section{References}

1. Cavalieri, S., S. Terzi, and M. Macchi, A Benchmarking Service for the evaluation and comparison of scheduling techniques. Computers in Industry Performance Measurement Special Issue, 2007. 58(7): p. 656-666.

2. Mishra, P. and P. Pandey, Simulation studies of flexible manufacturing systems using statistical design of experiments. Computers \& Industrial Engineering, 1989. 16(1): p. 65-74.

3. Mignon, D.J., S.J. Honkomp, and G.V. Reklaitis, A framework for investigating schedule robustness under uncertainty. Computers \& chemical engineering., 1995. 19: p. S615.

4. Cha, Y. and M. Jung, Satisfaction assessment of multi-objective schedules using neural fuzzy methodology. International Journal of Production Research, 2003. 41(8): p. 1831-1849.

5. Chan, F.T.S., H.K. Chan, and A. Kazerooni, A Fuzzy Multi-Criteria Decision-Making Technique for Evaluation of Scheduling Rules. The International 
journal, advanced manufacturing technology., 2002. 20: p. 103-113.

6. Brucker, P., Sceduling Algorithms. 1995, Berlin: Springer-Verlag.

7. Zhang, Y. and H. Chen, A knowledge-based dynamic job-scheduling in low-volume/high-variety manufacturing. Artificial intelligence in engineering., 1999. 13(3): p. 241.

8. Gupta, A.K. and A.I. Sivakumar, Job shop scheduling techniques in semiconductor manufacturing. International Journal of Advanced Manufacturing Technology, 2006. 27(11-12): p. 1163-12.

9. Ramasesh, R., Dynamic Job Shop Scheduling: A Survey of Simulation Research. Omega., 1990. 18(1): p. 43. 